\title{
Shall we screen for cardio-metabolic risks in methadone and buprenorphine treatment for opiate dependence?
}

\begin{abstract}
Recent evidence suggests that buprenorphine, the partial opiate receptor agonist, exhibits equal efficacy as methadone in reducing withdrawal and craving for opiates in opioid dependence. Very few studies examine the benefits of screening for combined cardiovascular and metabolic risk factors in methadone and buprenorphine maintenance options for opioid dependence. We explore the feasibility whether outcome maybe improved through developing cardio-metabolic screening strategy in treatment facilities for opioid addiction.
\end{abstract}

Keywords: methadone, buprenorphine metabolic cardiovascular risks
Volume 3 Issue I - 2017

\author{
Simon Chiu, ${ }^{1,6}$ Gamal Sadek, ${ }^{2}$ Larry \\ Lalone, ${ }^{6}$ Zack Cernovksy, ${ }^{\prime}$ Hana Raheb, ${ }^{6}$ \\ Kristen Terpstra, ${ }^{6}$ John Copen, ${ }^{3}$ Mariwan \\ Hunsi, ${ }^{4}$ Sidhu G, ${ }^{5}$ Andrew Sidhu, ${ }^{6,7}$ Autumn \\ Carriere, ${ }^{6,8}$ Zahra Khazaeipool, ${ }^{6}$ Christina \\ Chehade $^{6}$ \\ 'Department of Psychiatry, University of Western Ontario, UK \\ ${ }^{2}$ Private Methadone clinic, USA \\ ${ }^{3}$ Department of Psychiatry, University of British Columbia \\ Extended campus Victoria, USA \\ ${ }^{4}$ Consultant Psychiatrist, Northern Ontario Medical School \\ Thunder Bay, USA \\ ${ }^{5}$ Medical Director, Private Methadone clinic, USA \\ ${ }^{6}$ Lawson Health Research Institute, USA \\ ${ }^{7}$ National University of Ireland, Republic Ireland \\ ${ }^{8}$ Nipissing University North Bay, USA
}

Correspondence: Simon Chiu, Department of Psychiatry, University of Western Ontario, Parkwood Mental Health Care 550 Wellington St. London, Tel I-5 |9-455-5 I I0, Email schiu3207@rogers.com

Received: November 02, 2016 | Published: January 20, 2017
Abbreviations: MTT, methadone maintenance therapy; PET, positron emission tomography; DATOS, drug abuse treatment outcomes studies; NTORS, national treatment outcome research study; ATOS, australian treatment outcome study; QTC, quantum tunneling composite; TDP, torsades de pointes; BMI, body mass index.

\section{Introduction}

\section{Buprenorphine vs Methadone for opioid dependence}

Converging evidence suggests the efficacy of methadone maintenance therapy [MTT] for the treatment of opiate dependence. Methadone was seen as an opioid substitute for heroin dependence. Evidence suggests that MTT reduces significant harm and medical complications associated with intravenous drug abusers: hepatitis and HIV infection. ${ }^{1}$ A meta-analysis of MTT concludes that methadone is an efficacious maintenance intervention for the treatment of heroin dependence in terms of retaining clients longer and consistently in treatment and decreases the heroin use as compared with non-opioid replacement therapy. ${ }^{2}$ In North America [USA and Canada], within the past few years witnessed alarming rise in methadone-related deaths, along with marked increase in the misuse and abuse of prescribed opiates e.g. oxycontin for non-malignant pain syndrome and heroin abuse leading to fatal or near-fatal overdose. The unsuspected rise in methadone related deaths among severe opiate dependent clients with multiple relapses call into question the benefits/risk ratio. ${ }^{3}$
In contrast to methadone, buprenorphine behaves as a partial mu-opiate agonist and kappa antagonist, for the treatment of opiate dependence. Buprenorphine is favoured over pure opioid antagonist, naltrexone due to its partial opioid agonist properties. Intriguing enough, the Positron Emission Tomography [PET] study of mu-opioid receptor found that $16 \mathrm{mg}$ oral dosage of buprenorphine reduced mu-opioid receptor availability by $79-95 \%$. Opioid dependent subjects. ${ }^{4}$ The threshold blockade of opioid receptor is related to reduced craving for opioids. The mix agonist-antagonist activities of buprenorphine explain the efficacy of buprenorphine based treatment option in managing opioid dependence. The safety margins and the likelihood of abuse of buprenorphine have been highly favourable for buprenorphine, especially when formulated with naloxone as a sublingual formulation.

Marketed as [Suboxone]. ${ }^{5}$ The sublingual formulation of buprenorphine in combination with the opioid antagonist naloxone in a 4:1 ratio offers safeguard against medical diversion through intravenous self-administration. When buprenorphine/naloxone is taken sublingually as prescribed, the naloxone exerts no clinically significant effect, leaving the opioid agonist effects of buprenorphine to predominate. When buprenorphine/naloxone is parenterally administered in patients physically dependent on full agonist opioids, the opioid antagonism of naloxone causes withdrawal effects, thus reducing the abuse potential of the drug combination. Abuse-detergent and tamper-proof modes of delivery of buprenorphine represent new advances in opioid treatment. 
Harm reduction and avoidance, coupled with abstinence-based goal, has become the guiding principle underlying opioid treatment. Three major studies have documented the efficacy of methadone and buprenorphine in opioid dependence. Three longitudinal outcome studies: Drug Abuse Treatment Outcomes Studies [DATOS] in the United States, ${ }^{6}$ National Treatment Outcome Research Study [NTORS] in the United Kingdom, ${ }^{7}$ and the Australian Treatment Outcome Study $[\mathrm{ATOS}],{ }^{8}$ found that buprenorphine or methadone maintenance treatment can effectively reduce opioid abuse and misuse and minimize the harmful effects in the medical and psychosocial and medical arenas.

\section{Screening for cardio-vascular risks in opoid treatment}

The past few years witnessed alarming rise in methadone-related deaths along with marked increase in the misuse and abuse of prescribed opiates e.g. oxycontin for non-malignant pain syndrome. ${ }^{9}$ In reviewing the contributory factors, two patterns of methadonerelated deaths emerge. Early mortalities from methadone fatalities appear to be due to respiratory arrest during methadone induction: however, cardiac sudden death may also be an important factor. Methadone can induce QTC prolongation and the uncommon but potentially fatal ventricular arrhythmia, Torsades de Pointes [TDP]. ${ }^{10}$ A recent study found that QTC prolongation from baseline was statistically significant for methadone effect, ${ }^{11} 27 \%$ had QTC between $450 \mathrm{~ms}-500 \mathrm{~ms}$ and $6 \%$ had QTC $>500 \mathrm{~ms}$.

Onsite EKG screening to detect any QT prolongation [QTC $>450$ $\mathrm{ms}$ ] has been proposed by the panel of experts in cardiac safety in methadone prescribing. ${ }^{12}$ The panel recommends baseline EKG for all opiate dependence individuals being considered for MTT. If the corrected QTC interval is $>450 \mathrm{~ms}$ but less than $500 \mathrm{~ms}$ risk-benefit ratio should be discussed thoroughly with the potential MTT clients with more frequent monitoring. Contributory factors of prolongation of QTC interval: electrolyte imbalance, previous history of any angina, symptom history of syncope and seizure, drug-drug interaction e.g. certain types of antibiotics are to be included in the entry protocol. If the QTC interval is $>500 \mathrm{~ms}$, methadone dosage has been reduced or discontinued. Methadone dosage higher or at $120 \mathrm{mg}$ pod daily carried higher cardiac risk of significant QTC prolongation. The final common pathway of methadone-related ventricular arrhythmia, Torsades de Pointes, albeit uncommon, is potentially fatal. Buprenorphine, on the other hand, is less liable to induce QTC prolongation.

There have been limited studies on the prevalence of QTC prolongation among MTT clients. A Spanish cross-sectional study found $9.2 \%$ of MTT clients presented with QTC $>440 \mathrm{~ms}$ and $1.8 \%$ had QTC interval $>500 \mathrm{~ms} .{ }^{13}$ A US study found a higher prevalence of QTC prolongation in the cohort of MTT clinic registrants over 7 year- period: $27 \%$ had significant prolongation of QTC interval of $>450$ ms but less than $500 \mathrm{~ms} .{ }^{14}$ Web-based informatics paradigm can be the simplest measure to implement for EKG monitoring. In contrast, buprenorphine does not induce any QTC prolongation and confers wider cardiovascular safety margin for opioid dependence. ${ }^{5}$

Interestingly enough at the molecular level, the mechanism of methadone-induced QTC interval is thought to be correlated with the binding affinity to the voltage-gated potassium channel in the myocardium. ${ }^{15}$ Insights into the mechanistic details of QT prolongation and Torsades de pointes are gained from study of the congenital form of QTC prolongation estimated at $<1 / 10000$. Mutation alters the electrophysiological sequence of events of the cardiac cycle. In the cardiac cycle, potassium channel is responsible for returning the cardiac cycle to the resting phase before the next phase of myocardial cardiac cycle. Dysregulation in the repolarization cycle as regulated by inactivation of the sodium channel can result from a mutation in the single gene coding for a specific subtype of potassium channel: hERG [human either-a-go-go related gene] or coding for a variant of the sodium channel SCN5A, The binding affinity of opiate agonists and partial agonists for potassium channel can be evaluated with electrophysiological technique of measuring the changes in $\mathrm{K}+$ current through hERG channels expressed in HEK293 cells. The potassium channel coded by hERG gene has been localized on chromosome 7 and plays an important role in cardio-electric coupling. The binding affinity of methadone to the potassium channel is stereospecific, since the [S] isomer blocks the hERG current 3.5 fold more potently than the $[\mathrm{R}]$ isomer. $^{16}$

Very few studies have directed towards the issue of weight, diet and physical activity in opioid dependence. Our Medline search finds very few systematic studies on BMI [Body Mass Index] in methadone or buprenorphine treatment in opioid dependence. There is a paucity of data on the body composition: BMI, waist circumstance, relative proportion of body fat in the opiate dependence cohort. Two European studies suggested that the increase in weight gain reflecting improved nutritional status after entry to the MTT program. ${ }^{17,18}$ Our 1 yr follow up of MTT study reported that the means BMI shifted gradually towards the recent trend in body weight and BMI in the general population in that approximately $50 \%$ of the population are overweight as defined by $\mathrm{BMI}>25.0$ but less than 30.0. The recent renaissance of interest in screening and interventions to modify cardio-metabolic risk factors in opioid addiction has stimulated us to examine the cardiovascular risk factors along with metabolic risks in the area of diabetes and obesity prevention. We find very few published studies originating from Canada in this area. Our methadone/buprenorphine opioid treatment team in Ontario has recently found that the $43 \%$ of the sample of methadone clinic in subjects are overweight and obese: the results are summarized in the Figure $1 .{ }^{19}$

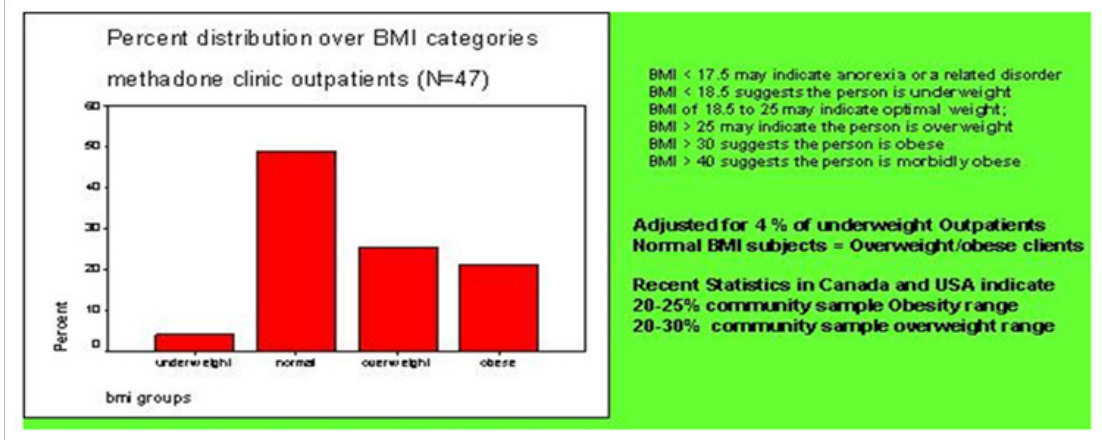

Figure I BMI distribution in Methadone Outpatients. ${ }^{19}$ 
A one -year follow up found a significant increase was noted in their mean BMI [increase from 24.1, $\mathrm{SD}=4.1$ to $26.3, \mathrm{SD}=4.8$ ], mean body mass [from $71.8 \mathrm{~kg}, \mathrm{SD}=14.4$ to $78.3 \mathrm{~kg}, \mathrm{SD}=15.9$ ], $\%$ fat [from $25.3 \%$ of total body mass, $\mathrm{SD}=10.0$ to $30.6 \%, \mathrm{SD}=9.3$ ] , and basal metabolic rate [from $1593.4, \mathrm{SD}=267.0$ to $1633.5, \mathrm{SD}=288.6$ ] and a significant decrease in $\%$ of muscle mass [from $71.0 \%$ of body mass, $\mathrm{SD}=9.6$ to $65.8 \%, \mathrm{SD}=8.9$ ], in $\%$ of water [from $52.0 \%$, $\mathrm{SD}=6.1$ to $48.8 \%, \mathrm{SD}=5.5$ ] and in $\%$ bone mass [from $3.7 \%, \mathrm{SD}=0.5$ to $3.5 \%, \mathrm{SD}=0.5] \cdot{ }^{20}$ We interpret the rise in $\mathrm{BMI}$ from normal value to overweight category to the increment in $\%$ of body fat, raising the issue of cardio-metabolic risk factors in opiate dependence. The change of subjective sleep quality is related to cocaine abuse, but unrelated to change in body composition. ${ }^{20}$

\section{Conclusion}

A recent Cochrane meta-analysis concludes that the strength of evidence does not support the effectiveness of QTC screening for preventing cardiovascular morbidity or mortality in methadone maintenance. In this respect buprenorphine has a definitive cardiovascular safety profile in the treatment of opioid dependence. Future endeavours should focus on the evaluating the benefits of developing QTC screening strategies and clarifying the relative benefits and harm reduction. ${ }^{21}$ Nevertheless, we have good reason to suggest that in the context of health promotion, an optimal screening strategy for identifying the cluster of cardio- and metabolic risks can improve outcome measures of opioid addiction. Primary care physicians are strategically positioned to order metabolic profile: fasting glucose, lipid and BMI [Body Mass Index] can be added to the screening panel for hepatitis and in certain high risk subgroups, HIV. Routine ECG alerting underlying cardiac arrhythmias and disease, and identifying QT-prolongation risks may prevent sudden cardiac death.

\section{Acknowledgements}

None.

\section{Conflict of interest}

The author declares no conflict of interest.

\section{References}

1. Ottawa (ON): Canadian Agency for Drugs and Technologies in Health Buprenorphine/Naloxone Versus Methadone for the Treatment of Opioid Dependence: A Review of Comparative Clinical Effectiveness, CostEffectiveness and Guidelines [Internet]. 2016.

2. Mattick RP, Breen C, Kimber J, et al. Methadone maintenance therapy versus no opioid replacement therapy for opioid dependence. Cochrane Database Syst Rev. 2009;8(3):CD0002209.

3. Jordan CO, Slater M, Kottke TE. Preventing Chronic Risk factors: rationale and feasibility. Medicine. 2008;44(10):745-750

4. Chugh SS, Socoteanu C, Reinier K, et al. A community-based evaluation of sudden death associated with therapeutic levels of methadone. Am J Med. 2008;121(1):66-71.
5. Mammen K, Bell J. The clinical efficacy and abuse potential of combination buprenorphine-naloxone in the treatment of opioid dependence. Expert Opin Pharmacother. 2009;10(15):2537-2544.

6. Hubbard RL, Craddock SG, Anderson J. Overview of 5-year follow-up outcomes in the Drug Abuse Treatment Outcome Studies (DATOS). $J$ Subst Abuse Treat. 2003;25(3):125-134.

7. Gossop M, Marsden J, Stewart D, et al. The National Treatment Outcome Research Study (NTORS): 4-5 year follow-up results. Addiction. 2003;98(3):291-303.

8. Teesson M, Mills K, Ross J, et al. The impact of treatment on 3 years outcome for heroin dependence: Findings from the Australian Treatment Outcome Study (ATOS). Addiction. 2008;103(1):80-88.

9. Modesto Lowe V, Brooks D, Petry N. Methadone deaths: risk factors in pain and addicted populations. J Gen Intern Med. 2010;25(4):305-309.

10. George S, Moreira K, Fapohunda M. Methadone and the heart: what the clinician needs to know. Curr Drug Abuse Rev. 2008;1(3):297-302.

11. Fareed A, Vayalapalli S, Byrd-Sellers J, et al. Onsite QTC interval screening for patients in methadone maintenance treatment. J Addict Dis. 2010;29(1):15-22.

12. Krantz MJ, Martin J, Stimmel B, et al. QTC interval screening in methadone treatment. Ann Intern Med. 2009;150(6):387-395.

13. Forseca F, Marti-Almor J, Pastor A, et al. Prevalence of long QTC interval in methadone maintenance patients. Drug Alcohol Depend. 2009;99(1-3):327-332.

14. Gongadze N, Kezeli T, Anelava N. Prolong QT interval and "torsades de pointes" associated with different group of drugs. Georgian Med News. 2007; 153:45-49.

15. Ansermot N, Albayrak O, Schlapter J, et al. Substitution of (R,S)methadone by ${ }^{\circledR}-$-methadone: impact on QTC interval. Arch Intern Med. 2010;170(6):529-536.

16. Okruhlica L, Slezakova S. Weight gain among the patients in methadone maintenance program as come-back to population norm. Cas Lek Cesk. 2008; $147(8): 426-430$.

17. Szpanowska Wohn A, Kolarzyk E, Pach D, et al. Nutritional status of opiate-dependent persons before and during methadone maintenance therapy. Przegl Lek. 2004;61(4):339-344.

18. Cernovsky Z, Sadek G, Chiu S. Relationships of body composition data to concurrent drug abuse in a methadone clinic. APA New Research Poster. Proceedings of Annual Meeting of American Psychiatric Association meeting Calf. 2016;5(1):e27587.

19. Cernovsky Z, Sadek G, Chiu S. Sleep Quality and Body Composition Variables in Opiate Substitution Treatment. Paper presented at the $12^{\text {th }}$ Annual Meeting of the International Society of Addiction Medicine. 2010 .

20. Pani PP, Trogu E, Maremmani I, et al. QTC interval screening for cardiac risk in methadone treatment of opioid dependence. Cochrane Database Syst Rev. 2013;6:CD008939.

21. Krantz MJ, Martin J, Stimmel B, et al. QTC interval screening in methadone treatment. Ann Intern Med. 2009;150(6):387-395. 